\title{
FRACTAL COMPRESSION TECHNIQUE FOR COLOR IMAGES USING VARIABLE BLOCK
}

\author{
Nisar Ahmed ${ }^{1}$ and Syed Abdul Sattar ${ }^{2}$ \\ ${ }^{1}$ Department of Electronics and Communication Engineering, Mewar University, India \\ ${ }^{2}$ Department of Electronics and Communication Engineering, Nawab Shah Alam Khan College of Engineering and Technology, India
}

\begin{abstract}
The main intention of Fractal Image Compression is to reduce the size of image and maintain good level of their reconstructed image. A major issue in Fractal Image Compression is decrease in image quality, compression ratio and PSNR. To overcome these issues we employ Fractal transformation with entropy coding. There are two phases in the proposed approach. In the first phase color images are separated into three RGB planes using variable range block size. In second phase by applying the inverse transform and iterative functions the image is restored. It is observed that the results are improving in fractal compression for both gray images as well as color images. In this work high CR and PSNR is observed compared to fixed block range and other existing methods. The proposed work yields better CR of 20 and high PSNR.
\end{abstract}

Keywords:

Fractal Image Compression, Variable Block Size, CR, PSNR

\section{INTRODUCTION}

Images are interpreted as accumulation of bits (a bit is a binary unit of information) and stored in digital media storages in the computer. These bits are denoted as pixels or points that form the picture elements. A human eye progresses large amount of information of approximately 8 million bits to create a single image. Profuse pixels constitute the formation of a reasonable quality image which, the eye arbitrates the 8 million bits from 'yes' and 'no' answers to develop the image progression. Redundancy of the pixels enriches the absorption capability of the eye to interpret the images into prominence, thus making them more apprehensible. This redundancy can be manipulated and reduced through algorithms for optimum storage and restored for recovery. An image can be alternated in many ways that are either not noticed by the human eye or do not contribute to the decay of the image. The current most famous method of image compression depend on elimination of high frequency wavelengths of the signal and storing only the low frequency components.

Fractal based image compression (FIC) technique exploit redundancy due to self-similarity properties in images to achieve compression [1]. A domain optimization technique in fractal image compression [2] deals with reducing complexity and increasing accuracy that is range and domain block alignment and matching. Fractal Image Compression is a separated image that can be a part of parts; the main motto of the fractal image compression is to reduce the redundancy or the image as well as image irrelevance to store [3]. Moreover image compression is a transformation called relative transformation. Fractal image compression is proposed in [4] based on Lossy compression principle which provides a very high compression ratio by completely eliminating the redundant information. The drawback of this technique is the degradation during reproduction of the image because of insufficient pixels for the filler sections comprising the redundant pixels. The image which is reconstructed contains degradation relative to the original image because the compression scheme completely neglects the redundant information and is more capable for achieving higher compression [5]. Fractal image compression (FIC) is based on the theory of Iterated Function System (IFS) which is used to represent the self-similarities of the images and is represented as a finite set of contraction mapping for each image that has the fixed point which is identical to the image itself [6]-[8]. Fractal image compression (FIC) seems to be one of the favorable methods of lossy image compression due to its advantages like resolution independence, high compression ratio, and fast decompression [9]. This technique was initially proposed by Jacquin and is based on the method of lossy compression. Here the images are considered to be fractals rather than pixels, which will be mainly suitable for natural images for encoding, analyzing with other image compression methods [10], [11] Suggested another system focused In the current scenario, fractal image compression plays a major role in research areas, it is one of the kind of modern image compression technique coefficients. Gohar and Habib [12] suggested in image compression two different processes is carried out namely encoding and decoding. In encoding section the original image takes as an input and after processing the compressed image will be its output but in decoding section the process is carried out in reversible order. In the event that the neighborhood heights are not fulfilled, the second phase of the calculation is completed keeping in mind the end goal to investigate further likenesses from the complete picture. Kharate and Patil [13] proposed fractal compression process through the self-similar properties in various fractions of images which relies on the fact that the image parts are often resembles other parts of the same image. Sekhar and Aruna [14] proposed a fractal is a rough or fragmented geometric shape that can be subdivided into parts, each of which is reduced/size copy of the whole. Khalil [15] found a major drawback in Fractal image compression (FIC), where encoding of fractals is very complex, compressing time is very high due to its complexity and consumption of time to search for the best matching block and more powerful. Anson [16] has recommended the IFIC using range block size. Barnsley and Hurd [17] have recommended applying the transformation iteratively on the random initial image and the result will be re-arranged as the original image. Banu [18] has proposed an efficient search of the domain pools for color image compression using Genetic Algorithm (GA). The proposed method reduces the coding process time and intensive computation tasks. Image quality factor, compression ratio and coding time are analyzed in this approach and comparative results are obtained but this method is suitable for only limited categories like facial, building and vegetable images. 


\section{FRACTAL COMPRESSION}

Fractal Image compression is one of the popular demanding research field in the current trend, mainly it deals with the images, video sequences, graphics, animations etc. Fractal compression is one of the kind of lossy image compression technique, here it will partition the images into blocks then perform some transformations and then compress it. Normally based on lossy compression, the compression ratio will be high but the quality of the images will not attain the expected results and there will be a loss of information's too. There are many technologies developed in different research areas to improve the image quality while compressing the images. In image compression consumption of time is more in encoding and decoding. There are some limitations existing too. This has motivated us to develop an efficient compression technique for color images to enhance high compression ratio and PSNR.

Various compression algorithms are used to reduce the size of the images without reducing the quality of images [19]. Algorithms like embedded zero wavelet algorithm, convergent algorithm, Fuzzy vector quantization algorithm etc. are used previously to compress the images with high quality [20].

\section{PROPOSED ALGORITHM}

The main objective of this research is to compress the image with high ratio and increase the PSNR of the encoding phase

Step 1: Divide color image into sub windows. Each sub window block is called R- block.

Step 2: For each R-block, find a Domain block, which most likely represents current R-block after a certain transform

Step 3: For every R-block, find D-block from D-pool which is most likely parallel to it.

Crop the D-block size to the size of R-block by Shrink operation and, mark the D-block as D'-block after Shrink operation is applied.

Transpose the $\mathrm{D}^{\prime}$ - block by using the following eight relative transformation equation matrices proposed by Jacquin. The eight relative transformations matrices are represented in the equations as follows,

Consider $T=\left[a_{i j}\right] n$ with $n=2$ where $1 \leq(i, j) \leq 2,\left[\begin{array}{ll}a_{11} & a_{12} \\ a_{21} & a_{22}\end{array}\right]$.

Relative transformations matrices can be represented with the following Eq.(1) to Eq.(8),

$$
\begin{gathered}
T_{0} \text { is } a_{i j}= \begin{cases}1 & \text { if } i=j \\
0 & \text { else }\end{cases} \\
T_{1} \text { is } a_{i j}=\left\{\begin{array}{cc}
(-1)^{2^{i}+j} & \text { if } i \neq j \\
0 & \text { else }
\end{array}\right. \\
T_{2} \text { is } a_{i j}=\left\{\begin{array}{cc}
-1 & \text { if } i=j \\
0 & \text { else }
\end{array}\right. \\
T_{3} \text { is } a_{i j}=\left\{\begin{array}{cc}
(-1)^{i+2^{j}} & \text { if } i \neq j \\
0 & \text { else }
\end{array}\right.
\end{gathered}
$$

$$
\begin{gathered}
T_{4} \text { is } a_{i j}=\left\{\begin{array}{cc}
(-1)^{i j} & \text { if } i=j \\
0 & \text { else }
\end{array}\right. \\
T_{5} \text { is } a_{i j}=\left\{\begin{array}{cc}
(-1)^{i i+1} & \text { if } i=j \\
0 & \text { else }
\end{array}\right. \\
T_{6} \text { is } a_{i j}=\left\{\begin{array}{cc}
(-1)^{i+j} & \text { if } i \neq j \\
0 & \text { else }
\end{array}\right. \\
T_{7} \text { is } a_{i j}=\left\{\begin{array}{cc}
1 & \text { if } i \pm j \\
0 & \text { else }
\end{array}\right.
\end{gathered}
$$

Above eight relative transformation blocks are generated for each $\mathrm{D}^{\prime}$-block and these blocks are composite new D-pool.

Compare each R-block with the blocks which are in $\mathrm{D}^{\prime}$ - pool and obtain nearly all like block. The parallelism can be measured with normal conflict MSE using the Eq.(9).

$$
M S E=\left\|X_{k} D_{k}+Y_{k} I-R_{k}\right\|
$$

where $X$ and $Y$ are the coefficients and should have the values for making the MSE minimum. The $X_{k}$ and $Y_{k}$ are represented in Eq.(10) and Eq.(11) respectively,

$$
\begin{gathered}
X_{k}=\frac{B^{2}\left\langle D_{k}, R_{k}\right\rangle-\left\langle D_{k}, I\right\rangle\left\langle R_{k}, I\right\rangle}{B^{2}\left\langle D_{k}, R_{k}\right\rangle-\left\langle D_{k}, I\right\rangle^{2}} \\
Y_{k}=\frac{\left\langle R_{k}, I\right\rangle-\left\langle D_{k}, I\right\rangle}{B^{2}} \\
W=\left\{D_{k}(x, y), T_{k}, X_{k}, Y_{k}\right\}
\end{gathered}
$$

where $R_{k}$ represents the relative transformation $W$ for each Rblock.

Fractal image coding, the original image is segmented into non-collaborating regions called range blocks and collaborating regions called domains blocks. For each range block affine transformations given in the Eq.(13) is used to match as best domain block.

$$
W_{i}\left[\begin{array}{c}
x \\
y \\
z
\end{array}\right]=\left[\begin{array}{ccc}
a_{i} & b_{i} & 0 \\
c_{i} & d_{i} & 0 \\
0 & 0 & s_{i}
\end{array}\right]\left[\begin{array}{l}
x \\
y \\
z
\end{array}\right]+\left[\begin{array}{c}
e_{i} \\
f_{i} \\
o_{i}
\end{array}\right]
$$

where $S_{i}$ controls the contrast and $O_{i}$ controls the brightness and $a_{i}, b_{i}, c_{i}, d_{i}, e_{i}, f_{i}$ denote the eight symmetries such as identity, reflection about mid-vertical and horizontal axis, first and second diagonal.

The Fig. 1 is the proposed fractal image compression.

1. Partition each plane image into non-overlapping blocks, called Range or R-block.

2. Select the maximum Range block of size $\left(R_{\max }\right)$ as 16 or 8 and minimum block of size $\left(R_{\min }\right)$ of 4 or 8 . R-blocks are compared with domains from the domain pool, which are double the range size.

3. A window size of $\alpha \times \alpha$ is slided on 3 images in $\alpha / 2$ or $\alpha / 4$. The pixels are averaged in groups to reduce to the size.

4. After partitioning and transformation, fractal encoding finds suitable candidate from all blocks to encode any particular R block. 
5. To increase encoding speed classification of sub-image into upper right, upper left, lower right and lower left quadrants is done as shown in Fig.2. It is shown that in any one of the three ways as canonical ordering [21].

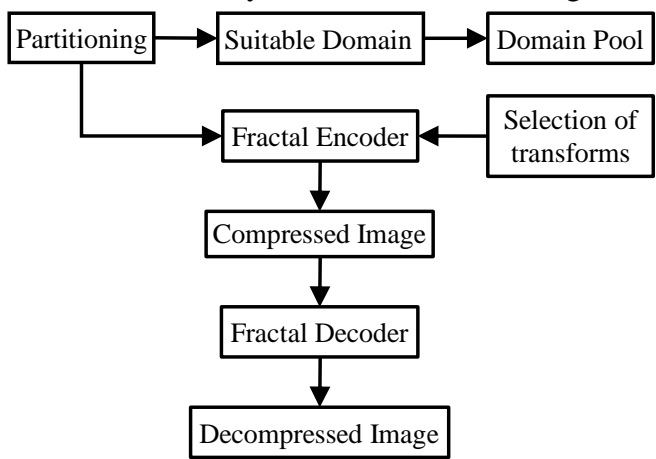

Fig.1. Proposed Fractal Compression Technique
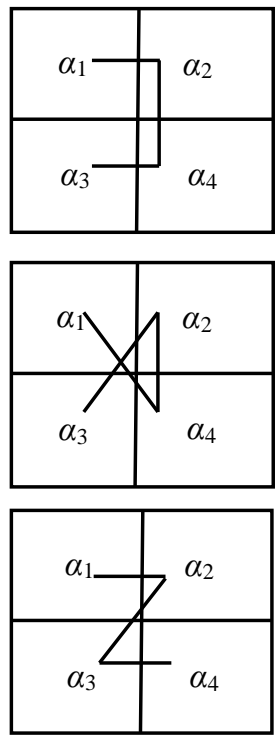

Fig.2. Arrangement of Sub Blocks Images

Classification Schemes are

- Major Class 1: $\alpha_{1}>\alpha_{2}>\alpha_{3}>\alpha_{4}$

- Major Class 1: $\alpha_{1}>\alpha_{4}>\alpha_{2}>\alpha_{3}$

- Major Class 1: $\alpha_{1}>\alpha_{2}>\alpha_{4}>\alpha_{3}$

Entropy of coding is used to achieve fractal compression for mapping the range block to the domain block in the coding process.

\section{RESULTS AND DISCUSSION}

To find the effectiveness of the proposed method by means of the experimental results. The Satellite urban and rural images are reduced to standard size $256 \times 256$. The standard Lena images of size $512 \times 512$ has been used for fractal compression analysis. The first phase consists of applying color and gray images to a set of five images. We have used five different test images in our experiment. In the final phase we have calculated the CR and PSNR with the resultant images. The imageries are subjected to fractal compression scheme by using the variable range block size for three cases namely,
a) $R_{\max }=16$ and $R_{\min }=4$
b) $R_{\text {max }}=16$ and $R_{\text {min }}=8$
c) $R_{\text {max }}=8$ and $R_{\text {min }}=4$,

Three different variable range methods are displayed in Table. 1 and Table.2, respectively. The novelty is, the proposed method is applied on both grey level and color images. The proposed method gives the comparable results when applied on both color and grey images. It is seen from Table. 1 that the CR and PSNR values derived from Lena, Satellite Rural, Rose, Satellite Urban and Bird imageries do not show significant difference between the CR and PSNR values. However, Table.1 clearly demonstrates that CR and PSNR values derived for the Lena and other imageries show maximum values for case (b) where the variable range block size $R_{\max }=16$ and $R_{\min }=8$. It is clearly seen from Table. 1 and Table. 2 shows for Color and Grey images values for CR and PSNR for all the types of imageries. The variable range block sizes shows higher CR and PSNR compared to fixed and other methods. This comparison is shown in Table.3, Table.4, Table.5, and Table.6, respectively.

Table.1. CR and PSNR of Test Color Images

\begin{tabular}{|c|c|c|c|c|c|c|}
\hline \multirow[t]{2}{*}{ Test Images } & \multicolumn{2}{|c|}{$\begin{array}{c}R_{\max }=16 \text { and } \\
R_{\min }=4\end{array}$} & \multicolumn{2}{|c|}{$\begin{array}{c}R_{\max }=16 \text { and } \\
R_{\min }=8\end{array}$} & \multicolumn{2}{|c|}{$\begin{array}{c}R_{\max }=8 \text { and } \\
R_{\min }=4\end{array}$} \\
\hline & CR & PSNR & $\mathbf{C R}$ & PSNR & CR & PSNR \\
\hline Lena Image & 2.99 & 27.88 & 15.45 & 20.16 & 3.16 & 30.15 \\
\hline $\begin{array}{c}\text { Satellite } \\
\text { Rural Image }\end{array}$ & 3.87 & 19.75 & 18.08 & 17.88 & 3.33 & 35.65 \\
\hline Rose Image & 4.44 & 27.01 & 17.85 & 20.11 & 4.02 & 35.66 \\
\hline $\begin{array}{c}\text { Satellite } \\
\text { Urban Image }\end{array}$ & 3.55 & 24.55 & 18.90 & 22.89 & 3.37 & 32.59 \\
\hline Bird Image & 3.68 & 18.88 & 17.45 & 24.95 & 3.11 & 31.79 \\
\hline
\end{tabular}

Table.2. CR and PSNR of Test Gray Images

\begin{tabular}{|c|c|c|c|c|c|c|}
\hline \multirow{2}{*}{$\begin{array}{c}\text { Test } \\
\text { Images }\end{array}$} & \multicolumn{2}{|c|}{$\begin{array}{c}R_{\max }=16 \text { and } \\
R_{\min }=4\end{array}$} & \multicolumn{2}{|c|}{$\begin{array}{c}R_{\max }=16 \text { and } \\
R_{\min }=8\end{array}$} & \multicolumn{2}{|c|}{$\begin{array}{c}R_{\max }=8 \text { and } \\
R_{\min }=4\end{array}$} \\
\hline & $\mathbf{C R}$ & PSNR & CR & PSNR & $\mathbf{C R}$ & PSNR \\
\hline Lena Image & 2.99 & 27.88 & 15.45 & 20.16 & 3.16 & 30.15 \\
\hline $\begin{array}{c}\text { Satellite } \\
\text { Rural Image }\end{array}$ & 3.87 & 19.75 & 18.08 & 17.88 & 3.33 & 35.65 \\
\hline Rose Image & 4.44 & 27.01 & 17.85 & 20.11 & 4.02 & 35.66 \\
\hline $\begin{array}{c}\text { Satellite } \\
\text { Urban } \\
\text { Image }\end{array}$ & 3.55 & 24.55 & 18.90 & 22.89 & 3.37 & 32.59 \\
\hline Bird Image & 3.68 & 18.88 & 17.45 & 24.95 & 3.11 & 31.79 \\
\hline
\end{tabular}

Variable range block size $R_{\max }=16$ and $R_{\min }=8$ are shown in Fig.3(a)-(b), Fig.4(a)-(b), Fig.5(a)-(b), Fig.6(a)-(b), Fig.7(a)-(b) and Fig.8(a)-(b). These are the reconstructed images for Color and Grey Lena, Rose, urban satellite, bird and rural satellite imageries. As seen in the figure and tables that the proposed IFIC method for the images of Lena, Bird, Satellite Rural, Rose and urban satellite gives significantly large CR values exhibits a very good quality of the reconstructed imageries. 


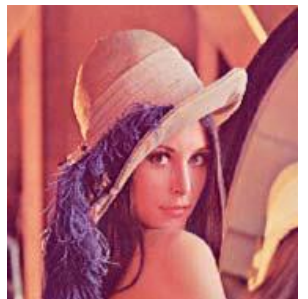

(a)

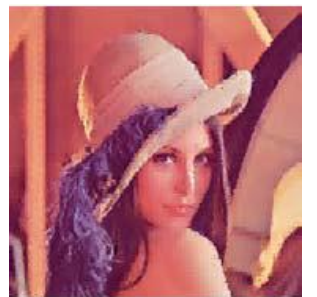

(b)
Fig.3. Results of Lena color image (a) Lena Image (b) reconstructed Lena image

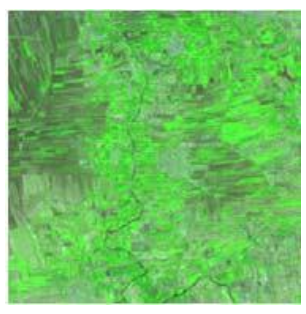

(a)

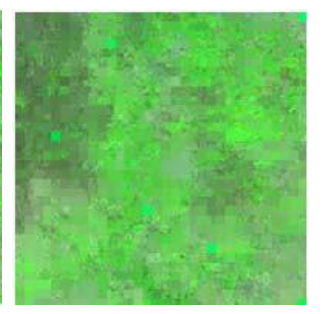

(b)
Fig.4. Results of Satellite Urban color image (a) original satellite urban Image (b) re-constructed satellite image

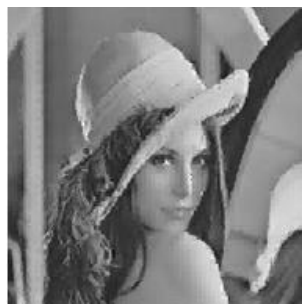

(a)

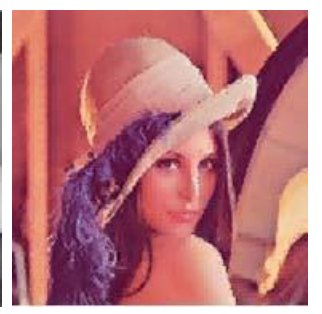

(b)
Fig.5. Results of Lena gray image (a) original lena gray Image (b) reconstructed lena image

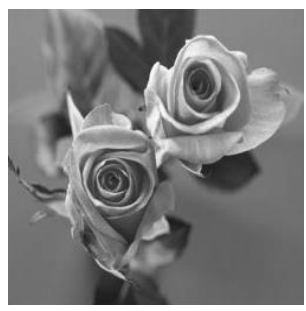

(a)

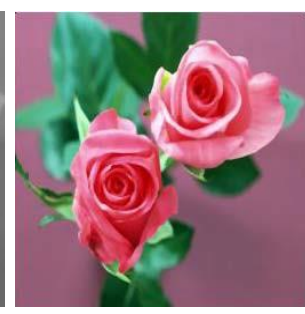

(b)
Fig.6. Results of Rose gray image (a) original rose gray Image (b) reconstructed rose image

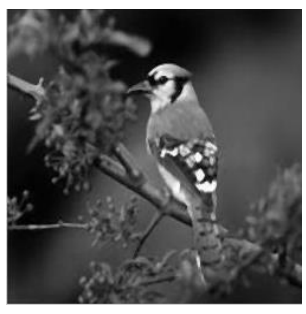

(a)

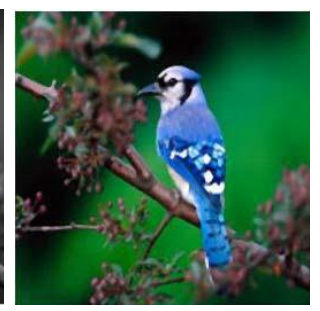

(b)
Fig.7. Results of Bird gray image (a) original gray bird Image (b) reconstructed bird image

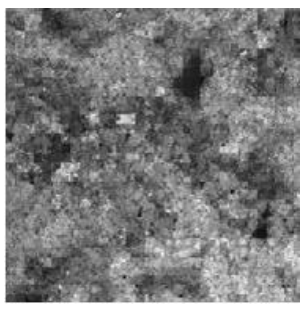

(a)

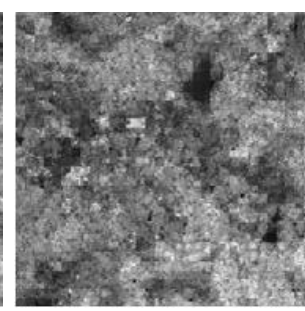

(b)
Fig.8. Results of satellite rural gray image (a) original gray satellite Image (b) reconstructed satellite image

\subsection{COMPARISON WITH OTHER EXISTING METHODS}

The present Fractal Image Compression [FIC] is compared with the other existing methods, like standard algorithm of quadtree technique [22], fractal with quadtree and DCT technique [23], and Fractal image compressions based on variable size range block method [24]. The CR and PSNR values obtained from the present method of variable range block size for test imageries are compared with the results of existing methods [22], [23], [24]. Standard algorithm of quadtree technique (SAQT), fractal with quadtree and DST technique (FQandDST), FIC based on variable size range block method (FICVRB), Proposed FIC in both color and gray level image with range block size $R_{\max }=16$ and $R_{\min }=8$ are comparatively shown in the Table. 3 to Table. 6.

Table.3. Comparison of CR with other available methods for color images

\begin{tabular}{|c|c|c|c|c|}
\hline Test Images & SAQT & $\begin{array}{c}\text { FQ and } \\
\text { DST }\end{array}$ & FIC VRB & FIC \\
\hline Lena Image & 10.01 & 14.44 & 13.0 & 17.53 \\
\hline $\begin{array}{c}\text { Satellite Rural } \\
\text { Image }\end{array}$ & 8.68 & 13.35 & 15.5 & 18.99 \\
\hline Rose Image & 8.32 & 12.56 & 15.4 & 18.13 \\
\hline $\begin{array}{c}\text { Satellite Urban } \\
\text { Image }\end{array}$ & 10.66 & 13.87 & 13.87 & 17.78 \\
\hline Bird Image & 9.99 & 14.98 & 16.56 & 16.55 \\
\hline
\end{tabular}

The comparison chart of CR and PSNR values for the proposed method and other existing methods in both case i.e. color and grey image when applied on test images i.e. Lena, Satellite rural image, Satellite urban image, Bird and Rose images are shown in Fig.9 to Fig.12.

Table.4. Comparison of CR with other available methods for grey images

\begin{tabular}{|c|c|c|c|c|}
\hline Test Images & SAQT & FQ and DST & FIC VRB & FIC \\
\hline Lena Image & 8.78 & 13.55 & 16.87 & 19.99 \\
\hline $\begin{array}{c}\text { Satellite Rural } \\
\text { Image }\end{array}$ & 9.77 & 13.33 & 15.75 & 18.89 \\
\hline Rose Image & 9.85 & 12.15 & 15.75 & 19.9 \\
\hline
\end{tabular}




\begin{tabular}{|c|c|c|c|c|}
\hline $\begin{array}{c}\text { Satellite Urban } \\
\text { Image }\end{array}$ & 9.15 & 13.03 & 17.71 & 18.53 \\
\hline Bird Image & 8.98 & 13.01 & 15.99 & 19.54 \\
\hline
\end{tabular}

Table.5. PSNR of the proposed method and other available methods of color images.

\begin{tabular}{|c|c|c|c|c|}
\hline Test Images & SAQT & FQ and DST & FIC VRB & FIC \\
\hline Lena Image & 24.89 & 29.45 & 18.55 & 30.33 \\
\hline $\begin{array}{c}\text { Satellite } \\
\text { Rural Image }\end{array}$ & 26.33 & 30.98 & 23.01 & 33.55 \\
\hline Rose Image & 26.08 & 32.01 & 21.50 & 35.55 \\
\hline $\begin{array}{c}\text { Satellite } \\
\text { Urban Image }\end{array}$ & 25 & 30 & 16 & 32 \\
\hline Bird Image & 26.45 & 31.67 & 23.80 & 33.11 \\
\hline
\end{tabular}

Table.6. PSNR of the proposed method and other available methods of gray level images

\begin{tabular}{|c|c|c|c|c|}
\hline Test Images & SAQT & FQ and DST & FIC VRB & FIC \\
\hline Lena Image & 23.13 & 29.88 & 26.5 & 32.25 \\
\hline $\begin{array}{c}\text { Satellite Rural } \\
\text { Image }\end{array}$ & 25.15 & 31.19 & 24.55 & 31.15 \\
\hline Rose Image & 24.33 & 29.10 & 18.55 & 32.01 \\
\hline $\begin{array}{c}\text { Satellite Urban } \\
\text { Image }\end{array}$ & 25.36 & 31.87 & 25.55 & 31.95 \\
\hline Bird Image & 25.15 & 30.48 & 23.76 & 32.78 \\
\hline
\end{tabular}

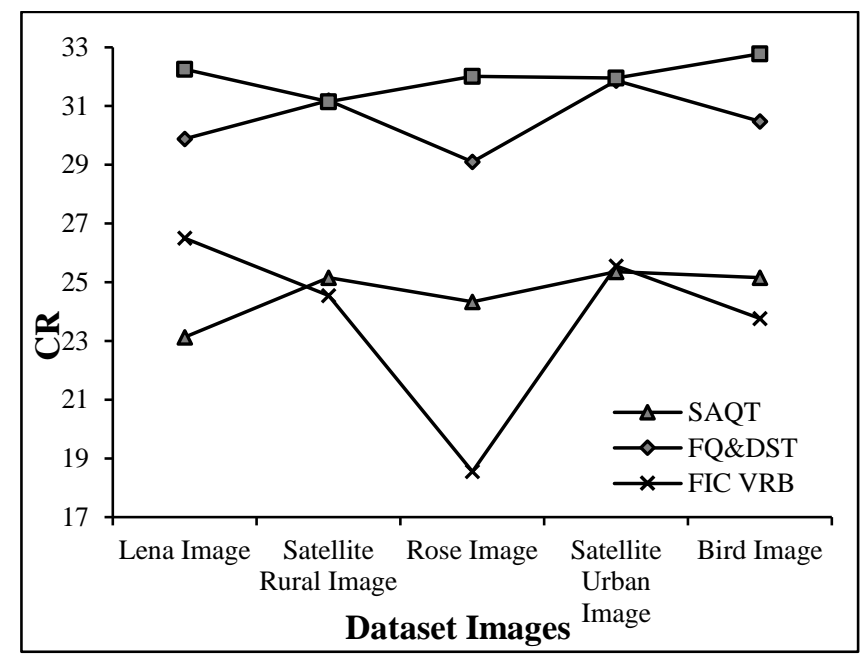

Fig.9. Comparison chart of CR of the proposed method with other existing methods when applied on color test images

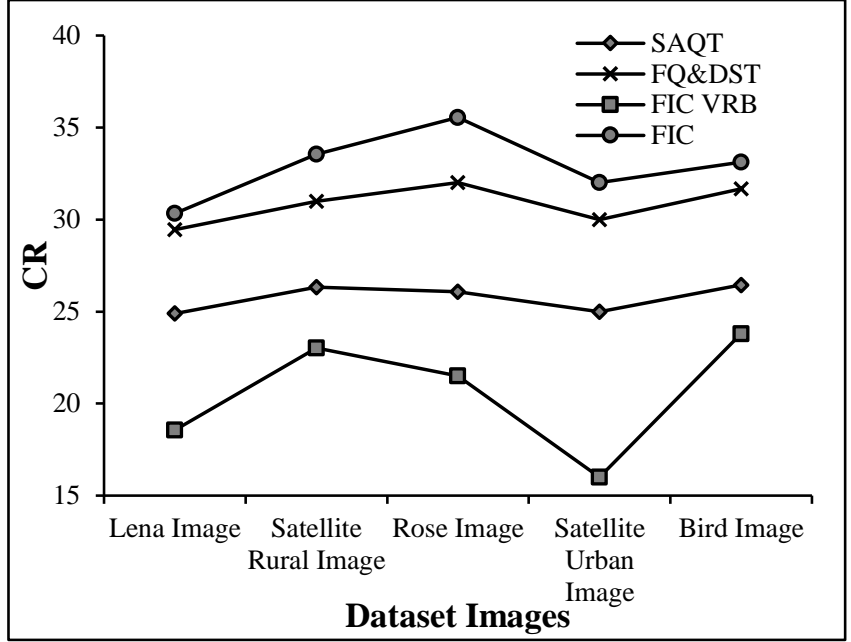

Fig.10. Existing methods when applied on gray test images Comparison chart of $\mathrm{CR}$ of the proposed method with other

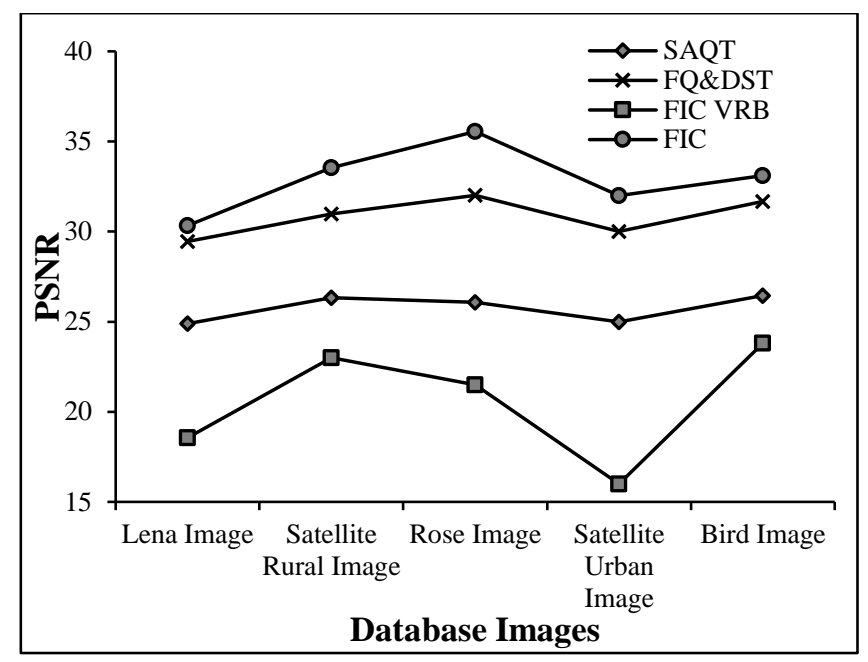

Fig.11. Comparison chart of PSNR of the proposed method with other existing methods when applied on color test images

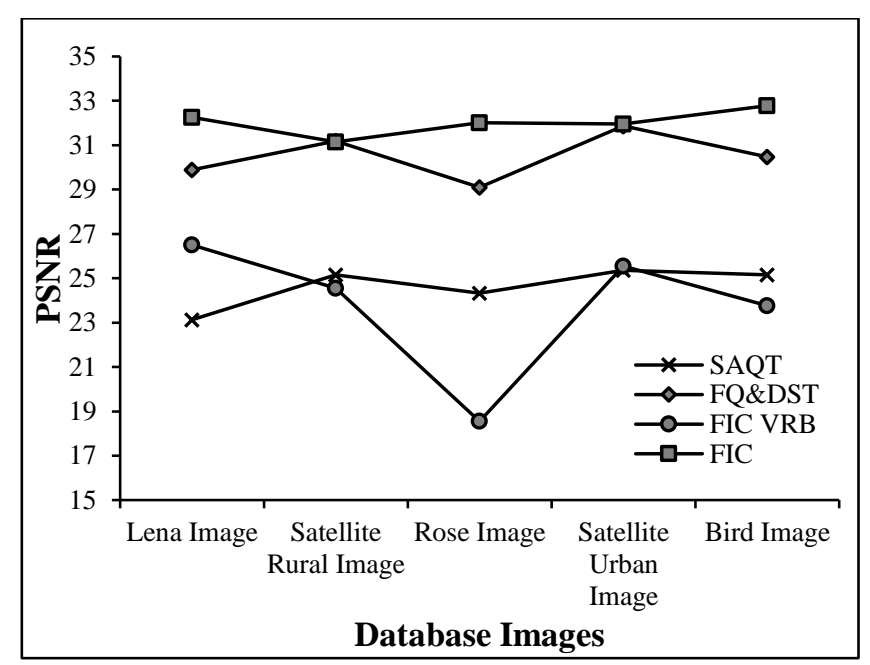

Fig.12. PSNR of the proposed method and other available methods of gray level images 


\section{CONCLUSION}

In the present paper, an experimental work is done to get an improved fractal image compression. The experimental results clearly depict that for the variable range block size of $R_{\max }=16$ and $R_{\min }=8$ a better result is yielded with good image quality and low computational cost. The uniqueness of the proposed method is, the RGB image is divided into three planes and using range block fractal concept it is compressed with reduced computational cost. When compare with the fixed block size method of fractal compression schemes, the present method exhibits higher compression ratio and PSNR values for all types of imageries. When the proposed method is applied on both color and gray scale images it gives approximately same CR and PSNR values. The proposed method when applied on different types of images like satellite image, standard color images and natural image, gives comparatively good results. The PSNR values obtained in this approach is always nearer to 30 .

\section{REFERENCES}

[1] Gagnpreet Kaur, Hitashi Hitashi and Gurdev Singh, "Performance Evaluation of Image Quality based on Fractal Image Compression", International Journal of Computers and Technology, Vol. 2, No.1, pp. 20-27, 2012.

[2] Zhuang Wu and Bixi Yan, "An Effective Fractal Image Compression Algorithm”, Proceedings of IEEE International Conference on Computer Application and System Modeling, pp. 139-143, 2010.

[3] Sumathi Poobal and G.Ravindran, "Analysis on the Effect of Tolerance Criteria in Fractal Image Compression", Proceedings of IEEE International Workshop on Imaging Systems and Techniques, pp. 119-124, 2005.

[4] A. Selim, M.M. Hadhoud, M.I. Dessouky and F.E. Abd El-Samie, "A Simplified Fractal Image Compression Algorithm”, Proceedings of IEEE International Conference on Computer Engineering and Systems, pp. 53-58, 2008.

[5] Dietmar Saupe, "Accelerating Fractal Image Compression by Multi-Dimensional Nearest Neighbor Search", Proceedings of IEEE Data Compression, pp. 222-231, 1995.

[6] Arnaud E. Jacquin, "Image Coding based on a Fractal Theory of Iterated Contractive Image Transformations", IEEE Transaction on Image Processing, Vol. 1, No. 1, pp. 18-30, 1992.

[7] M. Barnsley, "Fractals Everywhere", 2 $2^{\text {nd }}$ Edition, San Diego Academic Press, 1993.

[8] Y. Fisher, "Fractal Image Compression: Theory and Application", Springer, 1995.

[9] Brendt Wohlberg and Gerhard De Jager, "A Review of the Fractal Image Coding Literature", IEEE Transactions on Image Processing, Vol. 8, No. 12, pp. 1716-1729, 1999.

[10] Guojun Lu and Toon Lin Yew, "Applications of Partitioned Iterated Function Systems in Image and Video Compression", Journal of Visual Communication and Image Representation, Vol. 7, No. 2, pp. 144-154, 1996.
[11] Douda Sofia, Bagri Abdallah, Abdel Hakim and Amer Elimrani, "A Reduced Domain Pool based on DCT for a Fast Fractal Image Encoding", Electronic Letters on Computer Vision and Image Analysis, Vol. 10, No. 1, pp. 1123, 2011

[12] Vahdati Gohar, Khodadadi Habib, Yaghoobi Mahdi and Akbarzadeh-T Mohammad, "Fractal Image Compression Based on Spatial Correlation and Hybrid Particle Swarm Optimization with Genetic Algorithm", Proceedings of $22^{\text {nd }}$ International Conference on Software Technology and Engineering, pp. 134-138, 2010.

[13] G.K. Kharate and V.H. Patil, "Color Image Compression Based on Wavelet Packet Best Tree", International Journal of Computer Science Issues, Vol. 7, No. 2, pp. 31-35, 2010.

[14] D. Venkatasekhar and P. Aruna, "A Fast Fractal Image Compression using Huffman Coding", Asian Journal of Computer Science and Information Technology, Vol. 2, No. 9, pp. 272-275, 2012.

[15] M. Khalil, "Image Compression using New Entropy Coder", International Journal of Computer Theory and Engineering, Vol. 2, No. 1, pp. 39-42, 2010.

[16] Fractal Image Compression, Available at: http://www.math.psu.edu/tseng/class/Fractals.html.

[17] Michael Barnsley and Lyman Hurd, "Fractal Image Compression", AK Peters Limited, 1992.

[18] A.R. Nadira Banu Kamal and P. Priyanga, "Iteration Free Fractal Compression using Genetic Algorithm for Still Colour Images", ICTACT Journal on Image and Video Processing, Vol. 4, No. 3, pp. 785-790, 2014.

[19] Mohammed Ismail and S.M. Basha, "Improved Fractal Image Compression using Range Block Size", Proceedings of IEEE International Conference on Computer Graphics, Vision and Information Security, pp. 284-289, 2015.

[20] S.V. Veena Devi, A.G. Ananth, "Fractal Image Compression of Satellite Imageries using Variable Size of Range Block", Proceedings of IEEE International Conference on Signal and Image Processing Applications, pp. 172-175, 2013.

[21] Mario Polvere and Nappi Michele, "Speed-Up in Fractal Image Coding: Comparison of Methods", IEEE Transaction on Image Processing, Vol. 9, No. 6, pp. 10021009, 2000.

[22] A.H. Husseen, S.Sh. Mahmud and R.J. Mohammed, "Image Compression using Proposed Enhanced Run Length Encoding Algorithm", Ibn AL- Haitham Journal for Pure and Applied Science, Vol. 24, No. 1, pp. 18-25, 2011.

[23] K. Sharmila and K. Kuppusamy, "A New Color Image Compression Based on Fractal and Discrete Cosine Transform", International Journal of Engineering and Computer Science, Vol. 3, No. 7, pp. 7054-7057, 2014.

[24] S.V. Veenadevi and A.G. Ananth, "Fractal Image Compression of Satellite Color Imageries using Variable Size of Range Block", International Journal of Image Processing, Vol. 8, No. 1, pp. 1-8, 2014. 